\section{Vietnam Journal of Agricultural Sciences}

\title{
Investigating the Potential of Vietnamese Tea Seed Oil (Camellia sinensis O.Kuntze) for the Enhancement of Oxidative Stability in Vegetable Oils
}

\author{
Phan Thi Phuong Thao',2, Tran Thi Thu Hang ${ }^{2}$, Pham Le Nguyet \\ $\mathrm{Anh}^{3}$ \& Vu Hong Son ${ }^{1}$
}

${ }^{1}$ School of Food Biotechnology, Hanoi University of Science and Technology, Hanoi 112012, Vietnam

${ }^{2}$ Faculty of Food Science and Technology, Vietnam National University of Agriculture, Hanoi, 131000, Vietnam

${ }^{3}$ Faculty of Science, University of Sheffield, Sheffield S10 2TN, UK

\begin{abstract}
This study examined the effectiveness of different antioxidative compounds, namely $0.2 \%$ BHA (Butylated hydroxyanisole) + BHT (butylated hydroxytoluene), $0.03 \% \alpha-$ tocopherol, and $3 \%$ and $6 \%$ tea seed oil (TSO) on the oxidative stability of vegetable oils. Four commonly used oils, viz. rapeseed oil (RSO), peanut oil (PNO), sunflower oil (SFO), and soybean oil (SBO), were assessed by the Schall Oven test method and monitored during the 12-day preservation period under $60^{\circ} \mathrm{C}$. The total oxidation values (TOTOX) of the samples treated with $6 \%$ TSO were lower than those treated with $0.2 \%$ BHA+BHT. The results indicated the potential of TSO as a novel natural antioxidant for dietary vegetable oils. Our study also suggested that TSO could serve as an effective substitution for currently used synthetic antioxidants such as BHA and BHT.
\end{abstract}

\section{Keywords}

Tea seed oil, oxidative stability, oil preservation

\section{Introduction}

Oxidative stability during processing, storage, and cooking are some of the key determinants of the quality and shelf life of edible vegetable oils (Choe \& Min, 2006). Oxidation not only results in oil deterioration and production of off-flavor compounds but also negatively affects the nutritional values of dietary oils by breaking down essential fatty acids. Moreover, oxidation leads to the accumulation of toxic compounds that induce several health problems in humans including cancer, atherosclerosis, cardiovascular diseases, and allergies. Therefore, much effort has been made to inhibit oil oxidation during storage at low temperatures and cooking

Correspondence to

phanphuongthao@vnua.edu.vn 
at high temperatures. Current solutions are the inactivation of enzymes involved in oxidation, the addition of metal chelators, modifications to the packaging, and the addition of antioxidants.

Synthetic compounds including butylated hydroxyanisole (BHA), butylated hydroxytoluen (BHT), tert-butylhydroxyquinone (TBHQ), and propyl gallate (PG) are widely used antioxidants during oil processing (Merrill et al., 2008). These substances suppress oxidation in oils by scavenging free radicals and converting them into a more stable form (Choe \& Min, 2009). The amount of supplemented BHA and BHT is dependent on the lipid contents of the food products but should not exceed the suggested amount of 200 ppm (TCVN 7597:2013). A high dose of these phenolic compounds has been associated with health problems such as lung damage (BHT) or tumor development in the stomach (BHA) (Ito et al., 1985; Botterweck et al., 2000). Therefore, attempts have been made to exploit natural antioxidants in the replacement of synthetic compounds such as BHA and BHT to improve the shelf-life of vegetable oils. Several natural antioxidants, including ascorbyl palmitate (AP), tocopherol, catechin, and rosemary extract, etc., have been under investigation (Chu \& Hsu, 1999; Yanishlieva \& Marinova, 2001). Among these, tea seed oil (TSO) is a potential compound with an antioxidant activity that is as strong as or even stronger than that of BHT and vitamin E, respectively, at the same concentrations (Sahari et al., 2004). It was previously shown that the stability of TSO was the result of its low levels of polyunsaturated fatty acids such as linolenic and linoleic acids, and its antioxidant capacity relied on its phenolic compounds, especially EGCG, $\alpha$ - toccopherol, and tocotrienols (Sahari et al., 2004; Rajaei et al., 2008; Fazel et al., 2008; 2009).

According to a survey of Statista Research, the global consumptions of soybean oil (SBO), rapeseed oil (RSO), sunflower oil (SFO), and peanut oil (PNO) from 2013/2014 to 2019/2020 were 56.84 million tons, 27.77 million tons, 19.06 million tons, and 5.88 million tons, respectively (Shahbandeh, 2019). These widely consumed vegetable oils are sources of health- promoting and essential unsaturated fatty acids that cannot be synthesized by the human body (Orsavova et al., 2015). The health benefits of these oils are dependent on their fatty acid profiles and plant sources. SFO contains a high number of polyunsaturated fatty acids and a smaller number of monounsaturated fatty acids, together with a wide variety of natural antioxidants including $\alpha$ - tocopherol and the vitamins $\mathrm{A}, \mathrm{D}$, and $\mathrm{E}$ that can suppress oil oxidation (Choi et al., 2013; Guo et al., 2017). RSO is characterized by a similar fatty acid composition to that of SFO and a significant level of phenolic compounds such as $\alpha$-tocopherol (Jahreis \& Schäfer, 2011). PNO contains adverse fatty acid proportions, with a higher content of monounsaturated oleic acid than polyunsaturated linoleic acid, and the additional content of longer-chain fatty acids including arachidic (C20:0), behenic (C22:0), and lignoceric acid (C24:0) (Dean et al., 2011; Davis et al., 2016). In addition, polyunsaturated, saturated, and monounsaturated fatty acids required in human diets are also found in SBO in good proportions (de Almeida Chuffa et al., 2014). Although unsaturated fatty acids bring out several benefits for human hearts and bodies, their high levels result in accelerated oxidative susceptibility in vegetable oils, causing undesirable effects in the taste, aroma, and color, as well as in the chemical and nutrient values of the oils (Chen et al., 2014; Roszkowska et al., 2015; Maszewska et al., 2018; Redondo-Cuevas et al., 2018). Therefore, the addition of the appropriate type and quantity of antioxidants to these vegetable oils is crucial to improving their shelf-life and overall quality.

\section{Materials and Methods}

\section{Materials}

Pure soybean oil, rapeseed oil, peanut oil, and sunflower oil that were free of preservatives or additives were acquired from Cai Lan Vegetable Oil Company located at $649 \mathrm{Kim} \mathrm{Ma}$, Ngoc Khanh, Ba Dinh, Hanoi.

The antioxidants BHA and BHT were purchased from Sigma, Belgium; $\alpha$-tocopherol was purchased from Sigma-Aldrich, USA; and tea seed oil (Camellia sinensis $O$. Kuntze) was 
obtained from tea seeds harvested from mature tea plants of the "Trungdu" tea variety in Phu Tho province, Vietnam in December 2017.

The chemicals used in this experiment included acetic acid, chloroform, saturated KI, sodium thiosulfate $0.01 \mathrm{~N}, \quad \mathrm{p}$-anisidine, isooctane, and starch.

The equipment used in this experiment included a thermometer, ultrasound machine, freezer $\left(-80^{\circ} \mathrm{C}\right)$, JSSD microbiological cabinet, analytical scale Practum 224-1S (Germany), technical scale Ohaus (USA), and spectrophotometer UV-Vis Shimazu (Japan).

\section{Methods}

\section{Sample preparation and oxidation conditions}

The extraction process of tea seed oil is illustrated in Figure 1. Four vegetable oil samples were supplemented with different antioxidants and labeled as DTV.1, DTV.2, DTV.3, and DTV.4 as indicated in Table 1. A negative control (DTV) with no additional antioxidant was prepared for each tested oil. The samples were then transferred to $15-\mathrm{mL}$ falcon tubes and stored at $60^{\circ} \mathrm{C}$. Evaluations of both the negative control and tested samples were carried out on day 12 after the antioxidant supplementation. The analyzed parameters were peroxide, para-anisidine, and total oxidation (TOTOX) values.

\section{Analytical methods}

Analysis of the peroxide value: The peroxide values were determined according to TCVN 6121:2013. Approximately $0.5 \mathrm{~g}$ of each test sample was dissolved in $10 \mathrm{~mL}$ of an acetic acid:chloroform solution $(2: 1, \mathrm{v}: \mathrm{v}), 0.5 \mathrm{~mL}$ of freshly prepared saturated KI solution, and $15 \mathrm{~mL}$ of water. The mixture was titrated with a $0.01 \mathrm{~N}$ sodium thiosulfate $\left(\mathrm{Na}_{2} \mathrm{~S}_{2} \mathrm{O}_{3}\right)$ solution, with the starch solution being the indicator. The oil sample was replaced with 1 $\mathrm{ml}$ of water in the blank. The peroxide values (PV) (Meq $\mathrm{kg}^{-1}$ ) were calculated using the equation:

$$
\mathrm{PV}=\frac{\left(V-V_{0}\right) \times f \times N \times 1000}{m}
$$

where:

$\mathrm{PV}$ : peroxide value $\left(\mathrm{g} 100 \mathrm{~g}^{-1}\right)$;

$\mathrm{m}$ : mass of the oil sample (g);

$\mathrm{V}$ : volume of the $\mathrm{Na}_{2} \mathrm{~S}_{2} \mathrm{O}_{3}$ standard solution used in the test $(\mathrm{mL})$;

$\mathrm{V}_{0}$ : volume of the $\mathrm{Na}_{2} \mathrm{~S}_{2} \mathrm{O}_{3}$ standard solution used in the blank $(\mathrm{mL})$;

$\mathrm{N}$ : concentration of $\mathrm{Na}_{2} \mathrm{~S}_{2} \mathrm{O}_{3}\left(\mathrm{~mol} \mathrm{~L}^{-1}\right)$; and

$\mathrm{f}$ : concentration correction factor.

Analysis of para-anisidine value: The analyses were in accordance with TCVN 9670: 2013. The para-anisidine was weighed at $250 \mathrm{mg}$ and dissolved in acetic acid in a $50-\mathrm{mL}$ volumetric flask. Five milliliters of the sample solvent consisting of $0.2-0.3 \mathrm{~g}$ of the tested oils and $10 \mathrm{~mL}$ isooctane was supplemented with $1 \mathrm{ml}$ of para-anisidine solution and left to react in the dark (A1). One milliliter of the para-anisidine mixed with $5 \mathrm{~mL}$ isooctane instead of the sample

Table 1. Experimental design

\begin{tabular}{lcc}
\hline \multicolumn{1}{c}{ Formulas } & Preservative proportions & Description \\
\hline DTV & $0 \%$ & $100 \mathrm{~g}$ control vegetable oil \\
DTV.0 & $0 \%$ & $100 \mathrm{~g}$ control vegetable oil \\
DTV.1 & $0.015 \% \mathrm{BHA}$ & \\
& $0.005 \% \mathrm{BHT}$ & $99.98 \mathrm{~g}$ oil $+0.0015 \mathrm{~g} \mathrm{BHA}+0.005 \mathrm{~g} \mathrm{BHT}$ \\
DTV.2 & $0.03 \%$ & $99.97 \mathrm{~g}$ oil $+0.03 \mathrm{~g} \alpha-$ tocopherol \\
DTV.3 & $\alpha-$ tocopherol & $97 \mathrm{~g}$ oil $+3 \mathrm{~g}$ TSO \\
DTV.4 & $3 \%$ TSO & $97 \mathrm{~g}$ oil $+3 \mathrm{~g}$ TSO \\
\hline
\end{tabular}

Note: DTV represented four specific vegetable oils namely SBO, PNO, SFO, and RSO. 


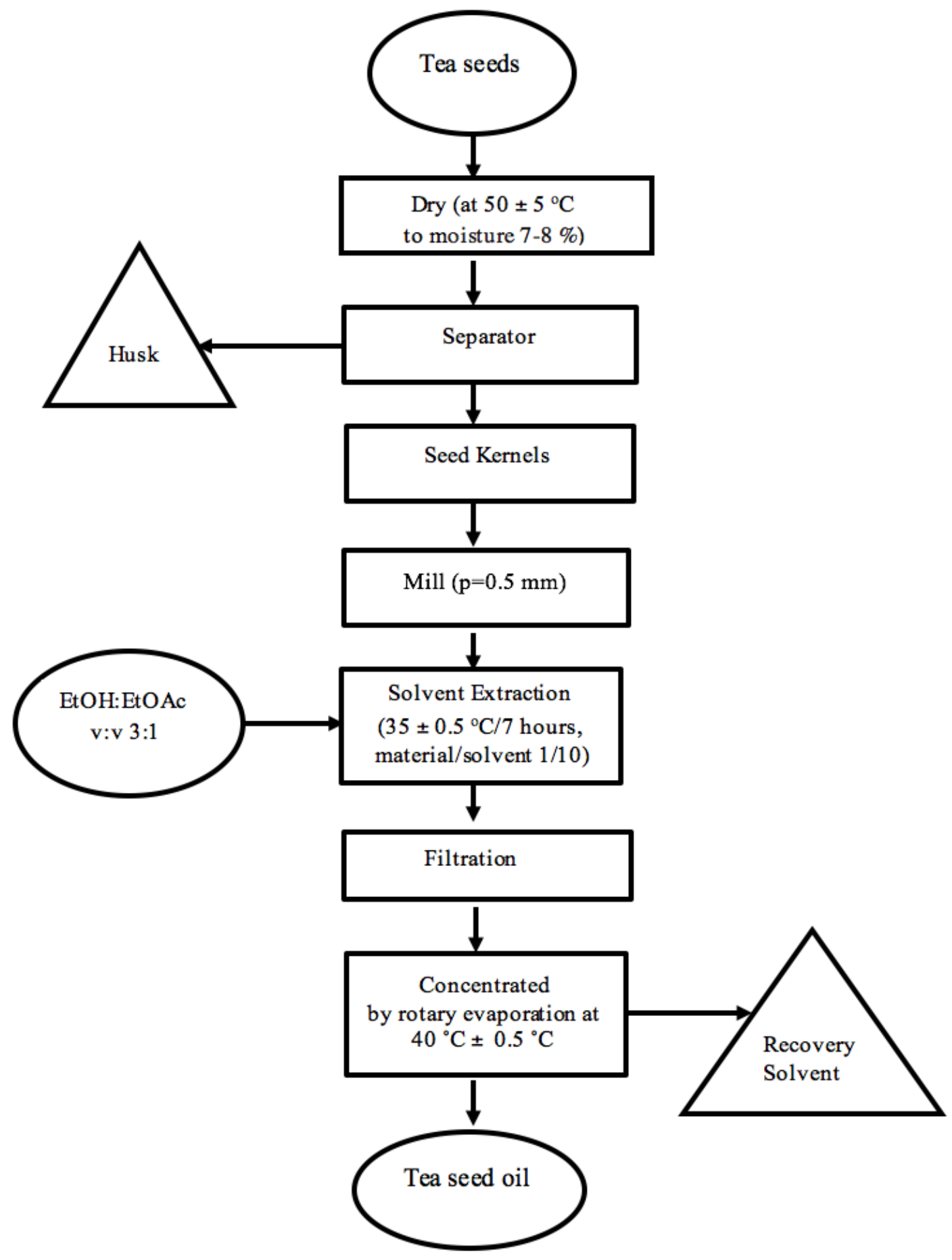

Figure 1. The extractive process of tea seed oil.

solvent was used as the blank (A2). An unreacted sample (A0) containing $5 \mathrm{~mL}$ of the sample solution and $1 \mathrm{~mL}$ acetic acid was prepared. After exactly 10 minutes, the absorbances of the three samples were determined at a wavelength of 350 nm. The para-anisidine values (P-Av) were calculated using this equation:

$$
\mathrm{P}-\mathrm{Av}=\frac{100 \times Q \times V}{m} \times 1.2 \times(A 1-A 2-A 0)
$$

where: 
P-Av: para-anisidine value (no unit);

Q: concentration of the sample used in the test $\left(\mathrm{g} \mathrm{mL}^{-1}\right)$;

$\mathrm{V}$ : volume of the dissolved sample $(\mathrm{mL})$;

$\mathrm{m}$ : mass of the oil sample (g);

1.2: correction factor for the dilution of the sample solutions with $1 \mathrm{~mL}$ para-anisidine reagent;

A1: absorbance of the reacted solution at 350nm;

A2: absorbance of the blank at $350 \mathrm{~nm}$; and

A0: absorbance of the unreacted solution at 350nm.

Analysis of Totox: The total oxidation value is the sum of the P-Av and twice the PV, and was calculated as below:

Totox $=\mathrm{P}-\mathrm{Av}+2 \mathrm{PV}$

where:

P-Av: para-anisidine value and

PV: peroxide value.

\section{Statistical analysis method}

All the results were presented as the mean of triplicate measurements \pm standard deviation. When applicable, the data were subjected to oneway analysis of variance (ANOVA) using the Minitab statistical package version 16 at $P<0.05$. The least significant difference (LSD) test was used in a mean separation where statistically significant differences were recorded.

\section{Results}

Effects of antioxidants on the peroxide values of vegetable oils after 12 days of storage

The peroxide value indicates the abundance of the primary oxidation product, hydroperoxide (Gordon, 2004). It has been widely used to monitor the deterioration of oil products. Therefore, we measured this value of the four tested oils with and without the addition of preservatives to evaluate the effectiveness of additives in enhancing the oxidative stability of the vegetable oils during storage. The four tested oils (DTV) had similar peroxide values before the antioxidant supplementation (Figure 2).

The peroxide values of the control samples (no antioxidant added) and tested samples (antioxidant added) showed no significant differences on day $0(P=0.05)$. Analyses on day 12 indicated that there were significant increases in the hydroperoxide levels in the pure vegetable oils $(P=0.05)$. Among the pure oils tested, the highest and lowest peroxide values were observed in pure SFO (91 Meq kg-1) and SBO (41 Meq kg-1), respectively (Figure 2). Our data also demonstrated that additional antioxidants effectively suppressed oil oxidation, hence resulting in lower peroxide values in the enriched oils than those of the pure oils after storage (Figure 2). The changes varied among the different types of antioxidants and vegetable oils tested. The peroxide values of the enriched SFO experienced the most drastic changes among those of the four vegetable oils. Among these preservatives, the addition of $6 \%$ TSO resulted in the highest reductions of peroxide values of all the tested oils, which were by $55 \%$ (PNO), $40 \%$ (SFO and RSO), and 25\% (SBO). The combination of the two common synthetic antioxidants, BHA and BHA, had the least impact on the peroxide values in these four edible oils, except for SFO.

\section{Effects of antioxidants on the para-anisidine values after 12 days of storage}

Unstable hydroperoxides decompose into base radicals, resulting in the formation of secondary products such as unsaturated aldehydes, non-volatile aldehydes, ketones, acids, esters, alcohol, and short-chain hydrocarbons at high temperatures (Gordon, 2004). The concentration of these compounds was determined by the para-anisidine value. Figure 3 shows the changes in the P-Av of the control oils and antioxidant supplemented formulas.

Similar to the primary oxidation products, the secondary oxidative compounds accumulated abundantly after 12 days when the oils were not 


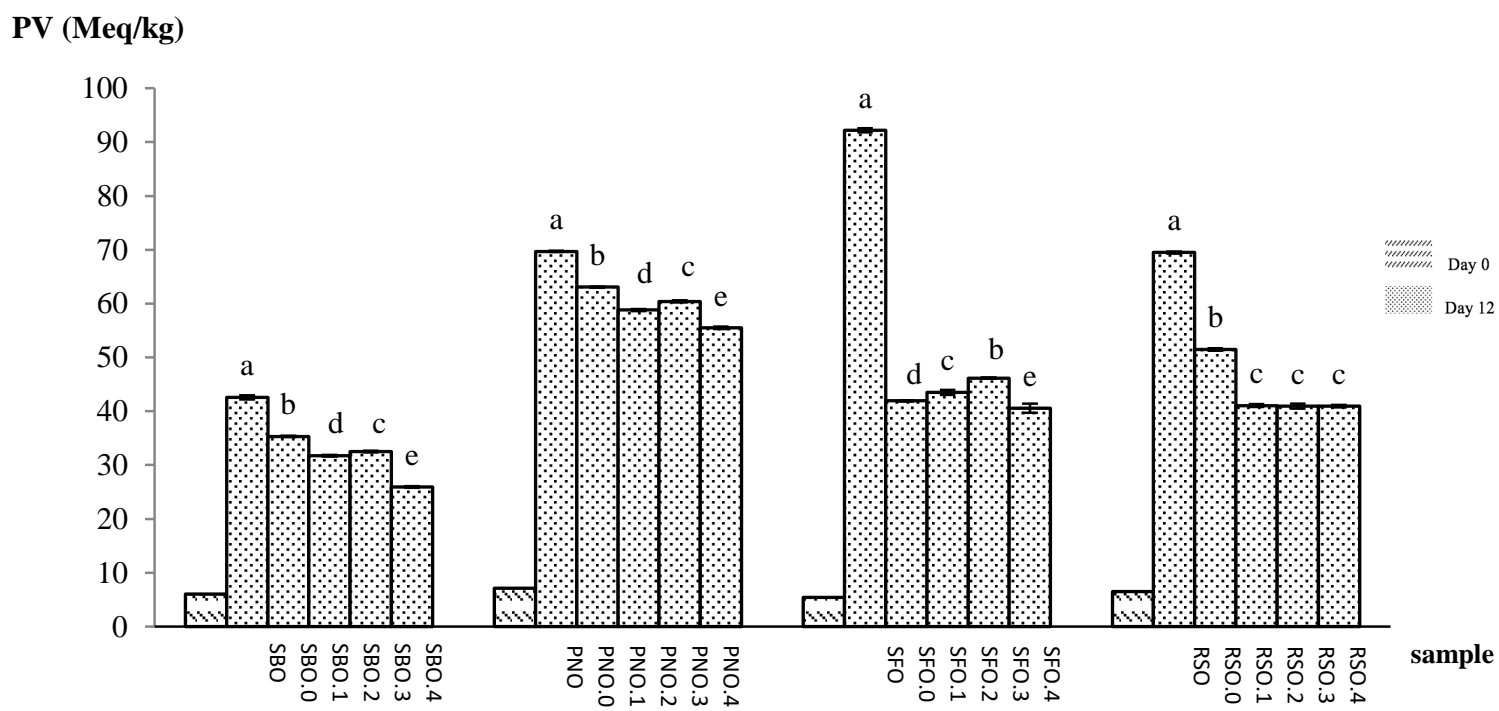

Note: The letters $a, b, c, d$, e, and f present significant differences at the $5 \%$ level of formulas in the specific oils.

Figure 2. The peroxide values of four vegetable oils with and without antioxidants after 12 days of storage: DTV (DTV = name of tested oils) columns represent the peroxide values for the pure tested oils before the addition of antioxidants (day 0 ). The peroxide values of the oils supplemented with no antioxidant, BHA+BHT mixture, $\alpha$-tocopherol, $3 \%$ TSO, and $6 \%$ TSO on day 12 are marked as DTV. 0, 1, 2, 3, and 4, respectively. SBO: Soybean oil, PNO: Peanut oil, SFO: Sunflower oil, and RSO: Rapeseed oil.

boosted with preservatives $(P=0.05)$ (Figure 3). The initial para-anisidine values varied among the pure vegetable oils, with the lowest and highest values observed in SBO $(\mathrm{P}-\mathrm{Av}=5)$ and SFO $(\mathrm{P}-\mathrm{Av}=14)$, respectively. Without additional preservatives, the hydroperoxide decomposition was most significant in pure SFO $(\mathrm{P}-\mathrm{Av}=38.5)$, followed by RSO $(\mathrm{P}-\mathrm{Av}=32)$, PNO (P-Av $=31)$, and SBO $(\mathrm{P}-\mathrm{Av}=18)$. The most drastic changes in the para-anisidine values were observed in RSO supplemented with all the tested antioxidants, BHA+BHT $(37.5 \%$ reduction), $\alpha$-tocopherol (70\% reduction), $3 \%$ TSO (65.5\% reduction), and $6 \%$ TSO $(68.8 \%$ reduction). TSO at a $6 \%$ concentration was the most effective antioxidant in terms of suppressing hydroperoxide decomposition in all four analyzed oils excluding RSO, which benefited slightly more from the addition of $\alpha$ tocopherol.

\section{The effects of antioxidants on the TOTOX values after 12 days of storage}

The total oxidation value of an oil is determined by adding the peroxide and paraanisidine values of the oil. It is an empirical measurement of the total oxidation products, and hence, indicates the overall oxidation state of oils during storage and handling (Symoniuk et al., 2017). After 12 days, pure SFO observed a ninefold increase in its TOTOX values, which was the highest increase in TOTOX values, followed by RSO (seven-fold), PNO (seven-fold), and SBO (five-fold) (Figure 4). All the analyzed additional antioxidants were shown to be effective in suppressing the oxidation rate in SFO. In agreement with the two previous parameters, the TOTOX values saw the highest reductions in all the vegetable oils boosted with $6 \%$ TSO. Supplementation with $6 \%$ TSO resulted in the highest reductions in the TOTOX values of SFO (58\%), followed by those of RSO (47\%), SBO (40\%), and PNO $(23.5 \%)$. In RSO, there was no significant difference in the TOTOX value changes resulting from the additions of $\alpha$-tocopherol, $3 \% \mathrm{TSO}$, and $6 \% \mathrm{TSO}$. 


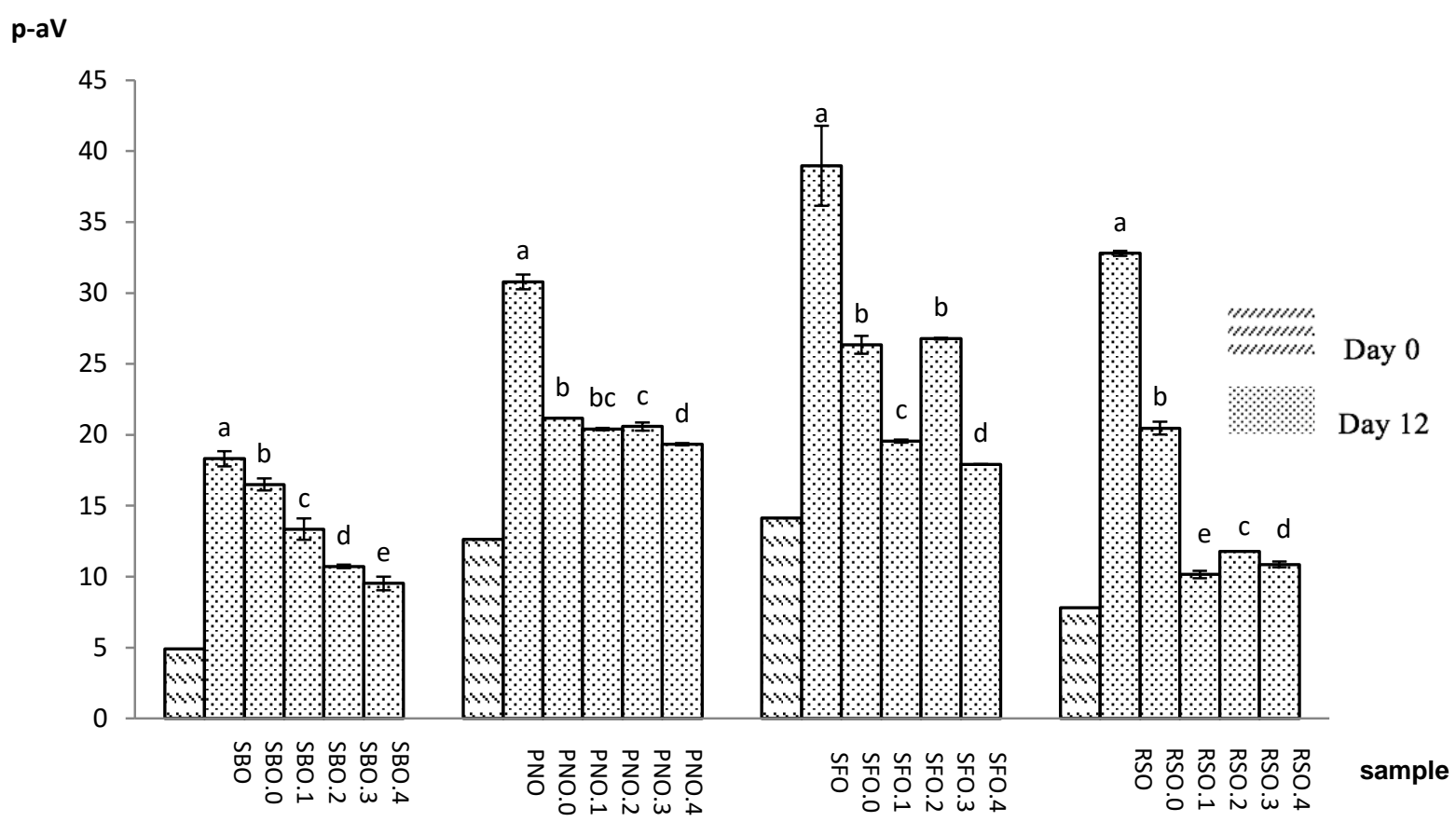

Note: The letters $a, b, c, d$, e, and f present significant differences at the $5 \%$ level of formulas in the specific oils.

Figure 3. The para-anisidine values of the four vegetable oils with and without antioxidants after 12 days of storage: SBO: Soybean oil; PNO: Peanut oil; SFO: Sunflower oil; and RSO: Rapeseed oil

\section{Discussion}

Due to the possible adverse effects of synthetic preservatives, many plants with novel sources of natural antioxidants have been extensively studied. TSO has become a potential candidate due to its high concentration of phenolic compounds ( $\alpha$-tocopherol, polyphenols) and carotenoids (Sahari et al., 2004). The autoxidation of oils results in increased free reactive radicals that can be removed by $\alpha$-tocopherol, polyphenols, and carotenoids via hydrogen transfer (Choe \& Min, 2005; 2009; Francenia Santos-Sánchez et al., 2019). Oil oxidation is assisted by prooxidative metals that minimize the activation energy of oxidation and catalyze radical chain reactions (Fereidoon \& Ying, 2010). Phenolic compounds such as flavonoids and phenolic acids act as metal chelators that inhibit the activity of metal ions (Rice-Evans et al., 1996). Another type of food oxidation, type II photosensitized oxidation, does not involve free radicals but requires nonradical singlet oxygen that directly reacts with unsaturated fats and oils (Choe \& Min, 2005). Singlet oxygen can be converted to its ground state, triplet oxygen, by carotenoids and phenolic compounds via energy transfer and charge transfer, respectively (Choe \& Min, 2009).

Our study evaluated the effectiveness of synthetic and natural antioxidants in improving the oxidative stability of vegetable oils based on three criteria: peroxide value (PV), paraanisidine value (P-Av), and total oxidation (TOTOX) value. Since PV measures the concentration of the unstable hydroperoxide, it does not accurately reflect the progress of oxidation or the effectiveness of antioxidants measures the concentration of the unstable 


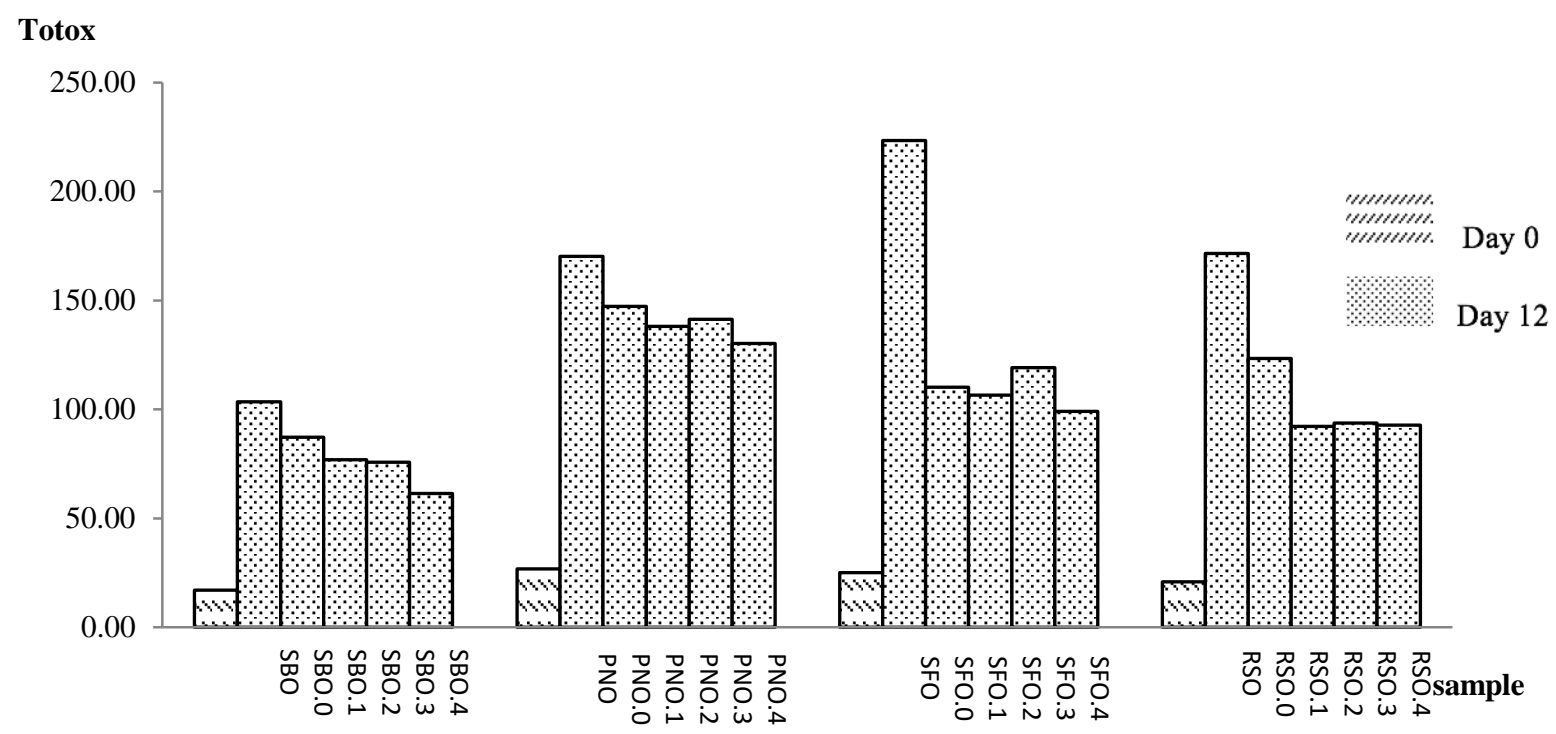

Figure 4. The TOTOX values of the four vegetable oils with and without antioxidants after 12 days of storage: SBO: Soybean oil, PNO: Peanut oil, SFO: Sunflower oil, and RSO: Rapeseed oil

hydroperoxide, it does not accurately reflect the progress of oxidation or the effectiveness of antioxidants (Gordon, 2004). Therefore, it must be used in conjunction with additional measurements such as P-Av, which measures the level of secondary oxidation products, and TOTOX, which indicates the total oxidation product concentration. The results from our study demonstrated that the inhibitory effect of TSO on oil oxidation was as strong as that of the synthetic compounds BHA and BHT. This harmonized with previous studies that illustrated the effectiveness of TSO in suppressing the oxidation susceptibility of edible vegetable and animal oils such as soybean and fish oils (Fazel et al., 2008; 2009; Prabsangob \& Benjakul, 2018). Our data also suggested that TSO at a higher concentration $(6 \%)$ was a more effective antioxidant than TSO at a lower concentration (3\%). Moreover, we observed that the TSOinduced oxidation suppression was more prominent in oils with higher levels of polyunsaturated fatty acids such as SFO than in those with lower levels of polyunsaturated fatty acids such as SBO and PNO. Unsaturated fatty acids provide several health benefits to the human body such as reducing the risk of inflammatory conditions, cardiovascular diseases, and cancers of the colon, heart, and prostate, and improving cognitive functions (Lunn \& Theobald, 2006). However, vegetable oils rich in unsaturated fatty acids, especially polyunsaturated ones with more double bonds, are more prone to oxidative rancidity than saturated fatty acid-rich oils, and hence, require optimal processing to preserve their nutrient contents while minimizing oil oxidative susceptibility. TSO was shown to be a potential preservative in these dietary oils. Future research should focus on analyzing the effect of TSO additions on the abundance of unsaturated fatty acids and other essential nutrients in vegetable oils.

\section{Conclusions}

The results from our study suggested that the four tested vegetable oils enriched with $6 \%$ TSO had comparable or higher oxidative stability than those supplemented with synthetic preservatives such as BHA and BHT. The addition of $6 \%$ TSO reduced the TOTOX values of pure SFO, RSO, SBO, and PNO by $58 \%, 47 \%, 40 \%$, and $23.5 \%$, respectively. It also resulted in the most 
significant reductions in the peroxide and paraanisidine values, although the changes varied among the four analyzed vegetable oils. The suppressing activity of other preservatives also varied among the four tested oils. The unequal effectiveness of the antioxidants may result from the physiochemical constituents of the oils and the interactions between additional and existing natural antioxidants in the vegetable oils. Further studies are required to unravel these complex relationships and maximize the efficiency of antioxidants during the preservation and usage of edible oils. Furthermore, the identification and quantification of possible anti-nutritional or toxic compounds in TSO are crucial to determine the usage and the appropriate dosage of TSO in dietary oils.

\section{References}

Botterweck A. A. M., Verhagen H., Goldbohm R. A., Kleinjans J. \& van den Brandt P. A. (2000). Intake of butylated hydroxyanisole and butylated hydroxytoluene and stomach cancer risk: results from analyses in the Netherlands Cohort Study. Food and Chemical Toxicology. 38(7): 599-605. DOI: 10.1016/S0278-6915(00)00042-9.

Chen X., Zhang Y., Zu Y., Yang L., Lu Q. \& Wang W. (2014). Antioxidant effects of rosemary extracts on sunflower oil compared with synthetic antioxidants. International Journal of Food Science and Technology. 49(2): 385-391. DOI: 10.1111/ijfs.12311.

Choe E. \& Min D. B. (2009). Mechanisms of Antioxidants in the Oxidation of Foods. Comprehensive Reviews in Food Science and Food Safety. DOI: 10.1111/j.15414337.2009.00085.x.

Choe E. \& Min D. B. (2006). Mechanisms and factors for edible oil oxidation. Comprehensive Reviews in Food Science and Food Safety. DOI: 10.1111/j.15414337.2006.00009.x.

Choe E. \& Min D. B. (2005). Chemistry and reactions of reactive oxygen species in foods. Journal of Food Science. Institute of Food Technologists. DOI: 10.1111/j.1365-2621.2005.tb08329.x.

Choi Y. S., Park K. S., Kim H. W., Hwang K. E., Song D. H., Choi M. S., Lee S. Y., Paik H. D. \& Kim C. J. (2013). Quality characteristics of reduced-fat frankfurters with pork fat replaced by sunflower seed oils and dietary fiber extracted from makgeolli lees. Meat Science. 93(3): 652-658. DOI: 10.1016/j.meatsci.2012.11.025.

Chu Y. H. \& Hsu H. F. (1999). Effects of antioxidants on peanut oil stability. Food Chemistry. 66(1): 29-34.
DOI: 10.1016/S0308-8146(98)00082-X.

Davis J. P., Price K. M., Dean L. L., Sweigart D. S., Cottonaro J. M. \& Sanders T. H. (2016). Peanut Oil Stability and Physical Properties Across a Range of Industrially Relevant Oleic Acid/Linoleic Acid Ratios. Peanut Science. 43(1): 1-11. DOI: 10.3146/0095-3679-43.1.1.

de Almeida Chuffa L. G., Vieira F. R., da Silva D. A. F. \& Franco D. M. (2014). Soybean seed oil: Nutritional composition, healthy benefits and commercial applications. In Seed Oil: Biological Properties, Health Benefits and Commercial Applications (pp. 124). Nova Science Publishers, Inc.

Dean Dr. L. L., Davis Dr. J. P. \& Sanders Dr. T. H. (2011). Groundnut (Peanut) Oil. In Vegetable Oils in Food Technology: Composition, Properties and Uses, Second Edition (pp. 226-242). John Wiley and Sons. DOI: 10.1002/97814-44339925.ch8.

Fazel M., Sahari M. A. \& Barzegar M. (2009). Comparison of tea and sesame seed oils as two natural antioxidants in a fish oil model system by radical scavenging activity. International Journal of Food Sciences and Nutrition. 60(7): 567-576. DOI: 10.3109/09637480801987625.

Fazel M., Sahari M. A. \& Barzegar M. (2008). Determination of Main Tea Seed Oil Antioxidants and their Effects on Common Kilka Oil. International Food Research Journal. 15(2): 209-217.

Fereidoon S. \& Ying Z. (2010). Lipid oxidation and improving the oxidative stability. Chemical Society Reviews. 39(11): 4067-4079. DOI: 10.1039/b922183m.

Francenia Santos-Sánchez N., Salas-Coronado R., Villanueva-Cañongo C. \& Hernández-Carlos B. (2019). Antioxidant Compounds and Their Antioxidant Mechanism. In Antioxidants. IntechOpen. DOI: $10.5772 /$ intechopen.85270.

Gordon M. H. (2004). Factors affecting lipid oxidation. In Understanding and Measuring the Shelf-Life of Food (pp. 128-141). Elsevier. DOI: 10.1533/9781855739024.1.128.

Guo S., Ge Y. \& Na Jom K. (2017). A review of phytochemistry, metabolite changes, and medicinal uses of the common sunflower seed and sprouts (Helianthus annuus L.). Chemistry Central Journal. BioMed Central Ltd. 11: Article number: 95. DOI: 10.1186/s13065-017-0328-7.

Ito N., Fukushima S. \& Tsuda H. (1985). Carcinogenicity and modification of the carcinogenic response by bha, bht, and other antioxidants. Critical Reviews in Toxicology. 15(2): 109-150. DOI: 10.3109/10408448509029322.

Jahreis G. \& Schäfer U. (2011). Rapeseed (Brassica napus) Oil and its Benefits for Human Health. In Nuts and Seeds in Health and Disease Prevention (pp. 967-974). Elsevier Inc. DOI: 10.1016/B978-0-12-375688- 
6.10114-8.

Lunn J. \& Theobald H. E. (2006). The health effects of dietary unsaturated fatty acids. Nutrition Bulletin. 31(3): 178-224. DOI: $10.1111 / \mathrm{j} .1467-$ 3010.2006.00571.x.

Maszewska M., Florowska A., Dłuzewska E., Wroniak M., Marciniak-Lukasiak K. \& Zbikowska A. (2018). Oxidative stability of selected edible oils. Molecules. 23(7). DOI: $10.3390 /$ molecules 23071746 .

Merrill L. I., Pike O. A., Ogden L. V. \& Dunn M. L. (2008). Oxidative stability of conventional and high-oleic vegetable oils with added antioxidants. JAOCS, Journal of the American Oil Chemists' Society. 85(8): 771-776. DOI: 10.1007/s11-746008-1256-4.

Orsavova J., Misurcova L., Vavra Ambrozova J., Vicha R. \& Mlcek J. (2015). Fatty acids composition of vegetable oils and its contribution to dietary energy intake and dependence of cardiovascular mortality on dietary intake of fatty acids. International Journal of Molecular Sciences. 16(6): 12871-12890. DOI: 10.3390/ijms 16-0612871.

Prabsangob N. \& Benjakul S. (2018). Enhancement of thermal stability of soybean oil by blending with tea seed oil. Emirates Journal of Food and Agriculture. 30(11): $\quad 968-977 . \quad$ DOI: 10.9755/ejfa.2018.v30.i11.1862.

Rajaei A., Barzegar M. \& Sahari M. A. (2008). Comparison of antioxidative effect of tea and sesame seed oils extracted by different methods. Journal of Agricultural Science and Technology. 10(4): 345-350.

Redondo-Cuevas L., Castellano G., Torrens F. \& Raikos V. (2018). Revealing the relationship between vegetable oil composition and oxidative stability: A multifactorial approach. Journal of Food Composition and Analysis. 66: 221-229. DOI: 10.1016/j.jfca.2017.12.027

Rice-Evans C. A., Miller N. J. \& Paganga G. (1996).
Structure-antioxidant activity relationships of flavonoids and phenolic acids. Free Radical Biology and Medicine. Elsevier Inc. DOI: 10.1016/08915849(95)02227-9.

Roszkowska B., Tańska M., Czaplicki S. \& Konopka I. (2015). Variation in the composition and oxidative stability of commercial rapeseed oils during their shelf life. European Journal of Lipid Science and Technology. 117(5): 673-683. DOI: 10.1002/ejlt.201400271.

Sahari M. A., Ataii D. \& Hamedi M. (2004). Characteristics of tea seed oil in comparison with sunflower and olive oils and its effect as a natural antioxidant. JAOCS, Journal of the American Oil Chemists' Society. 81(6): 585-588. DOI: 10.1007/s11746-006-0945-0.

Shahbandeh M. (2019). Consumption of vegetable oils worldwide from $2013 / 14$ to $2018 / 2019$ by oil type. Retrieved from https://www.statista.com/statistics/2$63937 /$ vegetable-oils-global-consumption/ on November 11, 2020.

Symoniuk E., Ratusz K. \& Krygier K. (2017). Oxidative stability and the chemical composition of market coldpressed linseed oil. European Journal of Lipid Science and Technology. 119(11). DOI: 10.1002/ejlt.201700055.

TCVN 6121:2010. Animal and vegetable fats and oils Determination of peroxide value - Iodometric (visual) endpoint determination (in Vietnamese).

TCVN 7597: 2013. Vegetable oils (in Vietnamese).

TCVN 9670:2013. Animal and vegetable fats and oils Determination of anisidine value (in Vietnamese).

Yanishlieva N. V. \& Marinova E. M. (2001). Stabilisation of edible oils with natural antioxidants. European Journal of Lipid Science and Technology. 103(11): 752-767. DOI: 10.1002/14389312(200111)103:11<752::AIDEJLT752>3.0.CO;2-0. 